\title{
Infezione da Bartonella henselae: caso clinico e supporto diagnostico
}

\author{
Salvatore Nisticò', Rosa Anna Leone', Pasquale Minchella', Giuseppe Ivan Potente', \\ Maria Teresa Cerminara', Caterina Mustaro', Bruno Romano², Maria Teresa Ruberto², \\ Angela Luciano' \\ ' U.O. Microbiologia e Virologia, PO Lamezia Terme, A S n. 6 di Lamezia Terme (CZ) \\ ${ }^{2}$ U.O. Pediatria, PO Soveria Mannelli - Azienda Sanitaria n. 6 di Lamezia Terme (CZ)
}

Key words: Cat scratch disease, Bartonella, test screening

Cat scratch disease: case report and quick antibodies test screening.

\section{SUMMARY}

Cat scratch disease is due to a bacterial infection sustained by Bartonella strains, transmitted to the human through the bite, scratch or lick of cats. We report a case about a young man who showed up to the Pediatrics outpatient clinic after he noticed a growing mass in his left armpit, preceded by malaise, fatigue and mild fever. The detection of the scar as a consequence of a cat scratch suggested the Bartonella infection diagnosis. Thus the patient had a blood test, the erythrocyte sedimentation velocity and specific antibodies assay: the measurement of serum Bartonella specific antibodies yelded high levels of IgM and IgG which confirmed the diagnosis. The patient was treated with a course of oral antibiotic, specifically Claritromicin $250 \mathrm{mg}$ tablets BID for two weeks. After 30 days the axillary nodal mass downsized. The serum immunoglobulin assay cut down the time required for the formulation of the causative diagnosis and allowed for a prompt and aimed antimicrobial therapy. Compared with the blood culture, the antibodies test screening is quicker and highly reliable.

\section{RIASSUNTO}

La malattia da graffio di gatto o Cat Scratch Disease (CSD) è una infezione sostenuta da microrganismi del genere Bartonella, trasmessi all'uomo attraverso lesioni provocate dal graffio dell'animale domestico. Il caso descritto riguarda un giovane che si è presentato all'ambulatorio dell'U.O. di Pediatria per la comparsa di una tumefazione localizzata al cavo ascellare sinistro, preceduta da malessere generale, astenia e febbricola.

La presenza all'esame obiettivo dell'esito cicatriziale di un graffio da gatto ha fatto sospettare un infezione da Bartonella. Sono stati pertanto eseguiti prelievi ematici per emocromo, VES e test sierologico per la ricerca di anticorpi anti-Bartonella; quest'ultimo ha dimostrato la presenza contemporanea di lgG ed IgM, confermando il sospetto etiologico.

Il paziente è stato trattato con terapia antibiotica a base di Claritromicina (cpr $250 \mathrm{mg} \times 2)$ per 15 giorni. Al controllo, dopo il ciclo di terapia, il paziente risultava clinicamente guarito.

La ricerca sierologica di immunoglobuline specifiche ha permesso di accorciare notevolmente i tempi di diagnosi etiologica ed ha permesso di instaurare una precoce e specifica terapia.

\section{INTRODUZIONE}

La malattia da graffio di gatto o Cat Scratch Disease (CSD) è un'infezione sostenuta da microrganismi del genere Bartonella (B. henselae, B. quintana, B. helizabethae e bacilliformis), trasmessi all'uomo attraverso lesioni provocate dal graffio dell'animale domestico. L'agente infettante è un bacillo Gram-negativo, aerobio obbligato, non ossidante e non fermentante (inerte), classificato precedentemente nel genere Rickettsie dal quale è stato successivamente separato in quanto non richiede, come le Rickettsie, substrati cellulari, ma può essere coltivato in vitro su idonei terreni arricchiti con sangue (umano, di coniglio, di cavallo); inoltre, studi effettuati sul DNA e sull'RNA ribosomiale 16S non hanno evidenziato alcuna correlazione tra $\mathrm{i}$ due generi. Il reservoir dei microrganismi è il gatto domestico e la trasmissione avviene attraverso il graffio o il morso dell'animale.

La lesione iniziale compare generalmente dopo 310 giorni nella sede del morso o del graffio sotto forma di una papula rosso-bruna, talvolta con tendenza all'ulcerazione, a cui segue dopo circa 1-2 settimane una adenite ad evoluzione suppurativa che interessa i linfonodi regionali. È spesso pre- 
sente febbricola (raramente la temperatura sale sopra i $38^{\circ} \mathrm{C}$ ) cefalea, malessere generale, nausea e vomito.

Il $5-10 \%$ dei casi può evolvere con gravi complicazioni quali la sindrome oculoghiandolare di Parinaud, granulomi epatosplenici, osteomielite, polmonite, arterite cerebrale, endocardite e porpora. È pertanto necessaria una diagnosi clinica tempestiva, supportata da indagini microbiologiche specifiche, al fine di instaurare idonea terapia antibiotica. Attualmente la diagnosi può essere posta direttamente con l'isolamento del germe (emocoltura) o indirettamente con test sierologici che ricercano anticorpi specifici IgG e/o IgM. L'emocoltura, tuttavia, ha tempi di risposta lunghi, legati alle caratteristiche di crescita lenta del batterio.

\section{Descrizione del caso clinico}

Un paziente di sesso maschile, di 17 anni, si è presentato all'osservazione dei medici dell'ambulatorio dell'Unità Operativa di Pediatria lamentando una tumefazione dolente a livello del cavo ascellare sinistro, malessere generale, astenia e febbricola. Ad una accurata indagine anamnestica il paziente riferiva di essere stato graffiato dal proprio gatto qualche settimana prima. All'esame obiettivo si apprezzava l'esito cicatriziale di una piccola ferita sul dorso della mano sinistra ed una tumefazione a livello del cavo ascellare sinistro per la presenza di un linfonodo di circa $4-5 \mathrm{~cm}$, duro, poco mobile e dolente alla palpazione. Sospettando una infezione da Bartonella sono stati eseguiti prelievi ematici per VES, PCR, emocromo e test sierologico per la ricerca di anticorpi anti-Bartonella nonché l'ecografia della regione ascellare come indagine strumentale. Per la ricerca di anticorpi IgG ed IgM anti-Bartonella sono stati utilizzati i kit diagnostici in immunofluorescenza indiretta (IFI) (Bartonella IFA IgG e Bartonella IFA IgM) della Ditta FOCUS, distribuiti in Italia dalla Ditta ALIFAX. Il kit per la ricerca delle $\operatorname{IgG}$ (sensibilità 95\% - specificità $88 \%$ ) contiene vetrini con 8 pozzetti a doppio substrato ciascuno: nel lato sinistro del pozzetto sono adese cellule Vero infettate da B. quintana, mentre nel lato destro sono adese cellule Vero infettate da $B$. henselae. I criteri interpretativi della metodica prevedono che un titolo di IgG $\geq 1: 256$ è significativo di infezione recente, indipendentemente dalla presenza di IgM; un titolo compreso tra 1:64 e 1:256, senza contemporanea positività di IgM, non è significativo ed è necessario testare un secondo prelievo effettuato a distanza di 15-20 giorni, per valutare una eventuale variazione del titolo anticorpale. Il kit per la ricerca delle IgM (sensibilità 95\% - specificità
$88 \%$ ) contiene vetrini con 8 pozzetti a doppio substrato ciascuno, caratterizzato da sospensioni batteriche di due tipi di Bartonella in matrice di sacco vitellino: nel lato sinistro del pozzetto sono adese sospensioni di $B$. quintana, mentre nel lato destro sospensioni di $B$. henselae. I criteri interpretativi della metodica prevedono che un titolo di $\operatorname{IgM} \geq 1: 20$ sia significativo di infezione in atto o recente.

I risultati degli esami di laboratorio eseguiti sul paziente giunto alla nostra osservazione, hanno rivelato un discreto aumento dei valori di VES e PCR nonchè un lieve aumento del globuli bianchi; l'ecografia della regione ascellare ha confermato la natura linfoghiandolare della tumefazione, con chiari segni di flogosi circostante.

Il risultato dei test per la ricerca di anticorpi antiBartonella ha dimostrato la presenza contemporanea di IgG (titolo $>1: 128$ ) e di IgM (titolo > $1: 64)$.

Avendo avuto conferma dai risultati delle indagini microbiologiche del sospetto diagnostico, il paziente veniva trattato con Claritromicina (cpr $250 \mathrm{mg} \times 2$ ) per 15 giorni. Al controllo, dopo il ciclo di terapia, il paziente risultava clinicamente guarito. Dopo circa un mese è stata effettuata una successiva indagine sierologica per la ricerca di anticorpi anti-Bartonella, che confermava la presenza di IgG specifiche (titolo $>1: 256$ ), mentre evidenziava la scomparsa delle IgM (titolo < 1:20). Purtroppo non è stato possibile eseguire l'emocoltura perché il paziente, al momento dell'osservazione, aveva già assunto antibiotici.

\section{DISCUSSIONE}

L'utilizzo della tecnica IFI per la ricerca di anticorpi anti-Bartonella può essere considerato un valido ausilio per una tempestiva diagnosi dell'infezione; infatti questo tipo di test ha tempi di esecuzione rapidi, avendo anche una buona sensibilità $(95 \%)$. Poiché l'emocoltura richiede tempi lunghi di incubazione e sistemi di identificazione non disponibili in tutti i laboratori di Microbiologia, per una corretta e tempestiva diagnosi etiologica della malattia da graffio di gatto può essere utilizzato in ogni laboratorio un sistema diagnostico basato su tecnica IFI.

\section{BIBLIOGRAFIA}

1. Anderson BE, Neuman NA. Bartonella spp as emerging human pathogens. Clin Microbiol Rev 1997; 10: 203-19.

2. Ciarrocchi G, Neri AM, Rondello G, Tocchini M, Gismondi P. Clinica e laboratorio nella diagnosi di Cat Scratch Disease: descrizione di cinque casi clinici. Microbiologia Medica 2002; 17 (3): 331-4.

3. Fabbi M, Vicari N, Tranquillo M, et al. Prevalence of 
Bartonella henselae in stray and domestic cats in different Italian areas: evaluation of the potential risk of transmission of Bartonella to humans. Parassitologia 2004; 46: 127-9.

4. Peter JB, Boyle M, Patnaik M, et al. Persistent generalized lymphadenopathy and non-Hodgkin's lymphoma in AIDS: association with Rochalimaea henselae infection. Clin and Diag Lab Immunology 1994; 1 (1): 115-6.

5. Zbinden R. Bartonella henselae - based indirect fluorescence assays are useful for diagnosis of Cat Scratch Disease. J Clin Microbiol 1998; 36 (12): 3741-2.

\section{Salvatore Nisticò}

U.O. Microbiologia e Virologia Azienda Sanitaria n. 6 Lamezia Terme Via A. Perugini, 188046 Lamezia Terme (CZ) Tel 0968/208761; Fax 0968/463113

E-mail: snistico1@virgilio.it 